\title{
Trauma penetrante com permanência de corpo estranho intraocular: diagnóstico e tratamento tardio
}

\author{
Penetrating trauma with permanence of an intraocular foreign body: diagnosis and late treatment \\ Traumatismo penetrante con permanencia de cuerpo extraño intraocular: diagnóstico y \\ tratamiento tardío
}

\begin{abstract}
Vinicio Tadeu da Silva Coelho ${ }^{1,2 *}$, Fabiano Costa Guedes ${ }^{2}$, José Luiz Braga Neto², Rafael Moreno Chaves $^{2}$, Cibelle Gonçalves Romero Pantel ${ }^{2}$, João Caetano Barbosa Duarte ${ }^{3}$, Marina Viana Melo ${ }^{3}$, Simone Soares da Silva ${ }^{3}$, César Barcellos Faiçal da Silveira ${ }^{4}$, Emilio Castellar Macedo Foureaux'.
\end{abstract}

\section{RESUMO}

Objetivo: Apresentar um caso de corpo estranho intraocular enfatizando o diagnóstico e tratamento tardio. Detalhamento do caso: Paciente, 71 anos, moradora de área rural, relatou perfuração do globo ocular esquerdo, socorrida em unidade de saúde onde realizaram sutura na córnea. Aproximadamente 131 dias, após sof rer a injúria foi encaminhada ao nosso serviço com queixa de baixa acuidade visual do Olho Esquerdo (OE). Ao exame oftalmológico verificou-se acuidade visual com correção em Olho Direito (OD) 20/20 e OE somente percepção luminosa. À biomicroscopia do OD sem alterações, no OE apresentava cicatriz corneana, leucoma central estendendo até a periferia temporal mostrando a porta de entrada do Corpo Estranho (CE), lesão e suturas na córnea, hiperemia conjuntival e pelo aspecto clínico caracterizando uma episclerite nodular. O cristalino apresentava opacidade central com pigmento de íris. À ultrassonografia do OE, evidenciou massa hiper-reflexiva, pico acústico alto, sugerindo Corpo Estranho Intraocular (CEIO). Considerações finais: Após o diagnóstico, a paciente foi encaminhada para cirurgias e remoção do CE. Assim, deve-se sempre anteceder ao ato terapêutico com exames de imagens capazes de permitirem a correta localização e dimensionamento do $\mathrm{CE}$, para garantir adequado planejamento terapêutico e cirúrgico, de forma que o prejuízo à visão seja reduzido.

Palavras-chave: Trauma penetrante, Corpo estranho intraocular, Diagnóstico e tratamento tardio.

\begin{abstract}
Objective: To present a case of an intraocular foreign body emphasizing the diagnosis and late treatment. Case details: Patient, 71 years old, resident of a rural area, reported perforation of the left eyeball, rescued at a health unit where they underwent suture in the cornea at the time. Approximately 131 days ago, after suffering the injury, she was referred to our service with a complaint of low visual acuity in the Left Eye (OI). Ophthalmological examination showed visual acuity with correction in Right Eye (OD) 20/20 and OI only light perception. Biomicroscopy of the OD showed no changes, in the Ol there was a corneal scar, central leukoma extending to the temporal periphery showing the entrance door of the Foreign Body (FB), lesion and sutures in the cornea, conjunctival hyperemia and, due to the clinical appearance, characterizing a nodular episcleritis. The lens had central opacity with iris pigment. On ultrasound of the Ol, she showed a hyper-reflective mass, high acoustic peak, suggesting Intraocular Foreign Body (IOFBs). Final considerations: After diagnosis, the patient was referred for surgery and removal of the FB. Thus, one should always precede the therapeutic act

\footnotetext{
1 Núcleo Médico de Oftalmologistas Ltda, Belo Horizonte - MG. *E-mail: viniciotadeu@yahoo.com.br

2 Faculdade de Saúde e Ecologia Humana (FASEH), Vespasiano - MG.

${ }^{3}$ Centro Universitário de Belo Horizonte (Uni-BH), Belo Horizonte - MG.

4 Faculdade Ciências Médicas de Minas Gerais (FCMMG), Belo Horizonte - MG.
} 
with imaging exams capable of allowing the correct location and dimensioning of the $\mathrm{FB}$, to ensure adequate therapeutic and surgical planning, so that the damage to vision is reduced.

Key words: Penetrating trauma, Intraocular foreign body, Diagnosis and late treatment.

\section{RESUMEN}

Objetivo: Presentar un caso de cuerpo extraño intraocular enf atizando en el diagnóstico y tratamiento tardío. Detalles del caso: Paciente de 71 años, residente de zona rural, refirió perforación del globo ocularizquierdo, rescatado en una unidad de salud donde en su momento se le realizó sutura en la córnea. Hace aproximadamente 131 días, luego de sufrir la lesión, fue derivada a nuestro servicio con queja de baja agudeza visual en el Ojo Izquierdo (OI). El examen oftalmológico mostró agudeza visual con corrección en Ojo Derecho (OD) 20/20 y Ol solo percepción de luz. La biomicroscopía del OD no mostró cambios, el OI presentaba cicatriz corneal, leucoma central con extensión a la periferia temporal mostrando la puerta de entrada del Cuerpo Extraño (CE), lesión corneal y suturas, hiperemia conjuntival y por el aspecto clínico caracterizando un epiescleritis nodular. El cristalino presentaba opacidad central con pigmento de iris. La ecografía del Ol mostró una masa hiperreflectante, de alto pico acústico, sugestiva de Cuerpo Extraño Intraocular (CEIO). Consideraciones finales: Posterior al diagnóstico, la paciente fue remitida para cirugía y remoción del CE. Así, siempre se debe anteceder al acto terapéutico con exámenes imagenológicos capaces de permitir la correcta localización y dimensionamiento del CE, para asegurar una adecuada planificación terapéutica y quirúrgica, de modo que se reduzca el daño a la visión.

Palabras clave: Traumatismo penetrante, Cuerpo extraño intraocular, Diagnóstico y tratamiento tardío.

\section{INTRODUÇÃO}

Trauma ocular penetrante é uma das principais causas de diminuição ou perda da acuidade visual, constituindo, assim, um enorme problema de saúde pública. O impacto social dessas lesões traduz-se em elevado custo hospitalar, af astamento do trabalho e gerando incapacidade visual permanente, atribuindo, uma carga economicamente negativa ao paciente e à sociedade (BHAGAT N, et al., 2011; RONG, AJ., 2019).

Traumas oculares com permanência de corpos estranhos intraoculares (CEIOs) referem-se a objetos estranhos que penetram e passam pela parede do globo ocular e se alojam no interior do olho. As lesões com CEIOs causam vários graus de dano aos tecidos oculares, sendo lesões graves e complexas devido à abertura do globo ocular. Nos países industrializados, as lesões com CEIOs estão entre as emergências oftalmológicas mais corriqueiras, causando danos à função visual de homens adultos jovens (LIANG Y, et al., 2021).

A presença de Corpos Estranhos Intraoculares (CEIOs) é responsável por 16\% de todos os traumas de globo aberto. A maioria dos CEIOs pós-traumáticos alojam no segmento posterior $69 \%$ e no segmento anterior em 31\% (LIU CC, et al., 2017). Como geralmente acontece nos traumas, a maioria dos acidentes com CEIOs são em homens jovens adultos e constituem entre 92 - 100\% dos pacientes (CHORAGIEWICZ T, et al., 2015).

As formas de agravo mais comuns, acompanhadas com permanência de CEIOs são a propulsão de fragmentos para o olho devido a marteladas, seguida por penetração de CE no olho devido a corte, exp losões, acidentes automobilísticos e outras causas respectivamente. A maior parte das lesões ocorre no ambiente de trabalho, e históricos médicos detalhados revelaram que quase todos os indivíduos não usavam óculos de proteção durante a realização de atividades de trabalho (LIANG Y, et al., 2021).

As manifestações oculares mais comuns são catarata traumática, hifema, hemorragia vítrea, descolamento de retina, vitreorretinopatia proliferativa e endoftalmite (LIANG Y, et al., 2021). Os descolamentos de retina pós-operatórios estão associados a CEIOs de segmento posterior e endoftalmite. Além disso, os olhos com locais de impacto dos CEIOs na retina têm um risco aumentado de descolamento no pós-operatório. Além das complicações principais, como descolamento de retina, hemorragia vítrea, catarata e endoftalmite, entre outras complicações (BOURKE L, et al., 2021). 
A maioria dos CE metálicos se aloja no segmento posterior do globo ocular. Por outro lado, partículas orgânicas e vegetais costumam se fixar no segmento anterior. Quando o trauma associa-se a retenção de um CEIO, as lesões são usualmente mais graves, pelo maior risco de inf ecção e toxicidades locais (ZHU L, et al., 2015).

Um CEIO ferroso pode levar à siderose bulbar, no qual, pode dar-se em até 18 dias após o trauma ocular. A instalação de partículas de ferro no olho resulta em siderose ocular, que abrange heterocromia da íris, midríase pupilar, formação de catarata, glaucoma secundário e degeneração pigmentar da retina (ZHU L, et al., 2015). O dano ocular é causado pela deposição de ferro liberado pelo CEIO como a ferritina em todo o citoplasma das células oculares, resultando em desorganização da retina com perda de segmentos dos receptores e células amácrinas colinérgicas, além do estresse oxidativo combinado com a liberação excessiva de glutamato e aumento do influxo de cálcio (LOPORCHIO D, et al., 2016).

Além dos sinais de um globo rompido, baixa Pressão Intraocular (PIO), câmara anterior rasa, contorno pupilar irregular, alguns sinais clínicos de um possível CEIO incluem pequenas feridas autovedantes, opacidade do cristalino, def eito de transiluminação e heterocromia da íris (UNVER YB, et al., 2009). Outras evidências de penetração do globo oculto incluem a hemorragia subconjuntival, quemose particularmente hemorrágica, teste de Seidel positivo e diminuição de movimentos extraoculares (UPSHAW JE, et al., 2008).

No momento do transporte para avaliação e após o exame até a cirurgia, o olho lesado deve ser protegido com um escudo rígido. Se um escudo não estiver disponível, outro dispositivo rígido (por exemplo, copo de isopor invertido) pode ser usado. O paciente deve começar a tomar antibióticos intravenosos de emergência se houver suspeita de lesão aberta do globo ocular. Os CEIOs representam uma ameaça significativa de infecção. Se houver suspeita de um CEIO, é importante garantir a cobertura para patógenos que são normalmente encontrados neste cenário, por exemplo, Bacillus, Clostridium e espécies de estafilococos. Se necessário, medicação anti-náusea pode ser usada para prevenir o vômito, que pode levar à expulsão do conteúdo intraocular (RATHOD R e WILLIAN FM, 2011; LOPORCHIO D, et al., 2016).

Os CEIOs podem alojar em locais que não se pode visualizar clinicamente, como na pars plicata ou alojados na íris posterior, mas que pode ser visualizado com biomicroscopia ultrassônica cuidadosamente realizada (KAUSHIK S, et al., 2008). A imagem ocular é uma ferramenta vital para o gerenciamento de CEIOs. Historicamente, ultrassonografia, radiografia, tomografia computado rizada, e ressonância magnética têm sido as ferramentas de imagem auxiliares utilizadas na detecção de CEIOs. A ferramenta de diagnóstico apropriada para visualização e localização depende da composição suspeita e da localização do CEIO (LOPORCHIO D, et al., 2016).

Em alguns casos, os CEIO oculto pode ser um olho com infecção intraocular que parece ser resistente à antibióticoterapia. Amostras para culturas devem ser coletadas se houver sinais e os sintomas indicarem a probabilidade de infecção intraocular. Uma nova análise por biomicroscopia de ultrassom, tomografia computadorizada ou ressonância magnética, se considerada segura, pode ser utilizada para avaliar esta possibilidade. Neste sentido, o médico deve sempre conjecturar que a endoftalmite está presente em todas as lesões oculares abertas até que prove o contrário. (BHAGAT N, et al., 2011).

A remoção cirúrgica do CEIO depende de vários fatores, incluindo o estado geral de saúde do paciente, a natureza da lesão e a composição do $\mathrm{CE}$. O reparo ocular com remoção hábil do CEIO é quase sempre indicado em pacientes com sinais clínicos de endoftalmite, exceto em casos de lesão simultânea com risco de vida que impediria a cirurgia oftalmológica. Se um oftalmologista não estiver disponível para realizar a cirurgia, pode ser mais prudente atrasar a remoção do CEIO, proteger o globo ocular e fazer admini stração de antibióticos intravítreos e sistêmicos. Assim, o paciente pode então ser encaminhado rapidamente ao especialista para tratamento definitivo de remoção do CE (LOPORCHIO D, et al., 2016; YEH S, et al., 2008).

O presente estudo teve como objetivo, reportar um caso clínico de trauma ocular, com permanência de corpo estranho no segmento posterior do olho por um longo período, revelando a importância do diagnóstico inicial e consequente acompanhamento da paciente. Além disso, propôs-se orientar para o manejo correto 
atentando para as características clínicas e os principais fatores de risco para o desenvolvimento de endoftalmite.

\section{DETALHAMENTO DO CASO}

Este estudo foi previamente submetido à Plataforma Brasil sobre o sistema CEP/Conep, sendo distribuíd o e aprovado pelo Comitê de ética e Pesquisa do Hospital Sofia Feldman, sob-registro CAAE $\mathrm{n}^{\circ}$ 45456821.9.0000.5132. Tramitado conforme atribuições contidas na carta circular $\mathrm{n}^{\circ} 166 / 2018$ CONEP/SECNS/MS de 12 de junho de 2018.

Paciente, 71 anos, sexo feminino, moradora de área rural, relatou perfuração do globo ocular esquerdo em maio/19, socorrida em unidade de saúde onde realizaram sutura na córnea. Aproximadamente 131 dias após sofrer a injúria ocular perfurante foi encaminhada ao nosso serviço ambulatorial com queixa de baixa acuidade visual do olho esquerdo. Ao exame oftalmológico verificou-se acuidade visual com correção em Olho Direito (OD) 20/20 e Olho Esquerdo (OE) somente percepção luminosa. À biomicroscopia de OD não apresentava lesão traumática. Em OE apresentava cicatriz corneana, leucoma central estendendo até a periferia temporal, mostrando a porta de entrada do Corpo Estranho (CE), lesão e suturas na córnea, hiperemia conjuntival e pelo aspecto clínico caracterizando uma episclerite. O cristalino apresentava opacidade central com pigmento de íris (Figura 1).

Figura 1 - Biomicroscopia óptica em lâmpada de fenda OE.

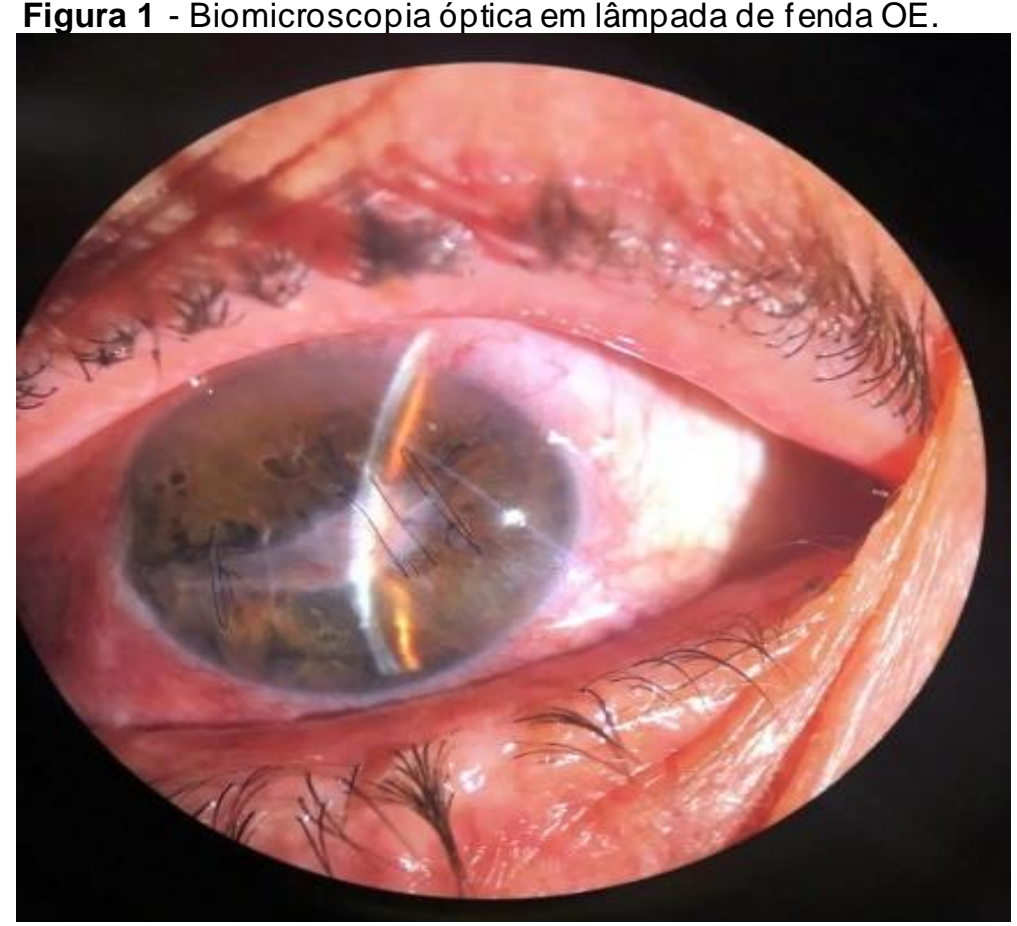

Nota: observa-se área de leucoma central estendendo até a periferia temporal mostrando porta de entrada do CE, lesão e suturas na córnea, hiperemia conjuntival com aspecto de episclerite nodular. Fonte: Coelho VTS, et al., 2022.

Ao exame de ultrassonografia ocular do OE, mostrou massa hiper-reflexiva, pico acústico alto, semelhante à parede ocular, evidenciando corpo estranho intraocular (CEIO) (Figura 2). Após análise do caso, a paciente foi encaminhada para cirurgias com indicação de transplante de córnea, facectomia, vitrectomia posterior e retirada de corpo estranho do OE. O prognóstico visual, neste caso, foi bastante reservado, e esteve diretamente associado a um prognóstico pós-operatório desfavorável, devido à permanência do CE no globo ocular por um longo período, pois apresentava acuidade visual inicial somente percepção luminosa, lesão perfurante e endoftalmite, conforme o modelo de pontuação de prognóstico para trauma ocular (KUHN F, et al., 2002). 


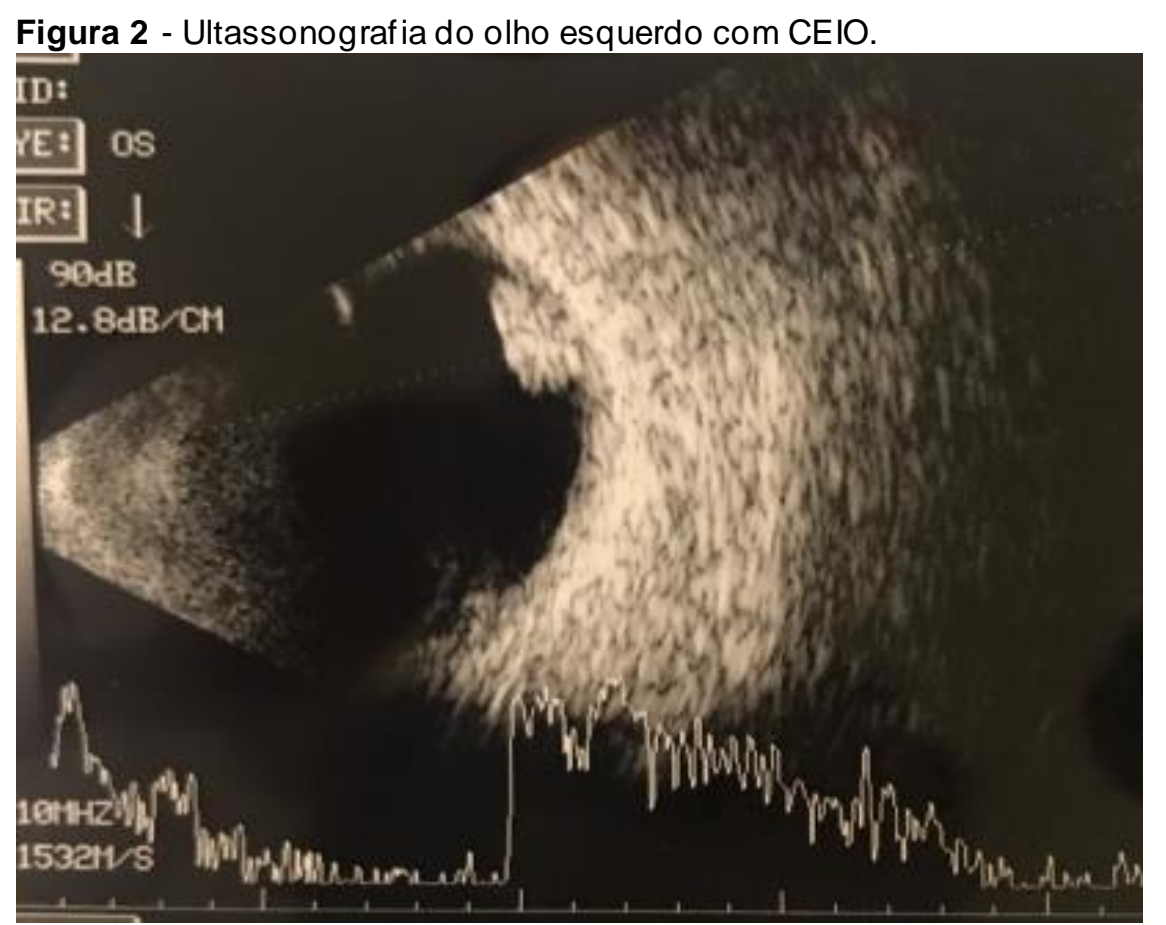

Nota: Imagem evidenciando massa hiper-reflexiva, pico acústico alto semelhante à parede ocular, revelando corpo estranho intraocular. Fonte: Coelho VTS, et al., 2022.

\section{DISCUSSÃO}

O trauma ocular, ainda é importante causa de perda visual, lesando o indivíduo e as companhias empregadoras, levando em consideração que grande parte dos pacientes é vítimas de incidentes ocupacionais (LIANG Y, et al., 2021). As características do CE como: tamanho, reatividade inflamatória e forma, bem como a velocidade de penetração, o trajeto e a localização final, são fatores que definem a amplitude da lesão inicial e as complicações futuras como exemplo a endoftalmite (LOPORCHIO D, et al., 2016).

Os CEIOs são uma emergência oftalmológica grave e uma causa comum de cegueira. Além de causar danos mecânicos aos tecidos oculares, os CEs retidos no olho podem causar danos químicos persistentes e infecção, af etando assim a função visual. Em casos graves, pode ocorrer cegueira ou atrofia do globo ocular, o que pode exigir a enucleação do olho af etado (LIANG Y, et al., 2021).

Após qualquer lesão traumática, infecciosa, cirúrgica ou relacionada à doença na córnea, o complexo processo de cicatrização do estroma da córnea é iniciado. Como o processo de cicatrização da ferida ocorre a partir dos ceratócitos nas proximidades da lesão, vai depender da extensão da lesão e, especialmente, se o epitélio da membrana basal (EMB) e / ou o epitélio da membrana de Descemet se regenera para o normal ou não. Se o epitélio cicatrizar e o EMB forem restaurados, a transparência é mantida ou pode ser restabelecida. Se o EMB não cicatrizar em tempo hábil, então os miofibroblastos maduros completam s eu desenvolvimento e a fibrose cicatricial é gerada (MARINO GK, et al., 2017; LASSANCE L, et al., 2018; WILSON SE., 2020).

O processo de cicatrização da córnea não ocorreu de forma ordenada e no tempo fis iológico necessário para obter-se uma restauração da membrana basal e assim manter a transparência da córnea. Para lesões mais graves com danos extensos, a regeneração retardada das membranas basais leva à penetração grandes quantidades de componentes da matriz extracelular desordenada para produzir fibrose cicatricial (TORRICELLI AAM, et al., 2013).

A catarata traumática ocorre devido a forças mecânicas e químicas sobre o cristalino, que alteram sua forma e suas funções metabólicas. Alterações como aumento do cristalino, podem provocar a ruptura da 
capsula cristaliniana ou zonular, além de alterações metabólicas e teciduais, levando a opacificação (SHAH M, et al., 2013). Com o exame de biomicroscopia, diagnosticou-se, catarata traumática, que se caracterizo u por opacificação do cristalino unilateral e seu prognóstico relacionou-se ao acometimento das estruturas oculares adjacentes.

Os achados clínicos, caracterizando sinais clássicos de siderose ocular, incluem heterocromia da íris, midríase pupilar, formação de catarata e degeneração pigmentar da retina e hiperemia do disco óptico, esse quadro, pode se desenvolver em olhos que abrigam corpos estranhos intraoculares contendo ferro, (LOPORCHIO D, et al., 2016). Importante ressaltar que a progressão para siderose ocular seja provável, pois, o corpo estranho foi localizado no segmento posterior. A causa da visão diminuída da paciente foi provavelmente, devido à opacidade subcapsular anterior, corroborando com a hipótese de siderose ocular posterior.

Os exames de observação direta, na maioria das vezes, são o que bastam para o diagnóstico, entretanto, exames complementares de imagem, radiografias e ultrassons, são ferramentas importantes no auxilio do diagnóstico (LOPORCHIO D, et al., 2016). A ultrassonografia possibilitou diagnosticar o corpo estranho no segmento posterior e inferir sua a natureza metálica, esclarecer o seu trajeto e determinar a sua localização, facilitando, posteriormente, a remoção cirúrgica, evitando causar maiores lesões ao globo ocular.

A episclerite é uma inflamação não granulomatosa da rede vascular episcleral. Nódulo de tecido inflamatório pode estar presente em cerca de $20 \%$ dos casos, sendo esta condição denominada de episclerite nodular e, quando ausente, é mais apropriadamente denominada episclerite difusa. Esse processo inflamatório agudo envolve a ativação de células imunes residentes, incluindo linfócitos e macrófagos. Uma vez ativados, eles liberam mediadores inflamatórios causando vasodilatação, aumento da permeabilidade vascular e migração de mais glóbulos brancos e macrófagos (SALAMA AMR, et al., 2018).

No nosso estudo, a paciente apresentou hiperemia conjuntival, caracterizando um quadro de episclerite nodular devido à endoftalmite. A episclerite geralmente é uma doença benigna e autolimitada, mas não deve ser banalizada, pois pode estar associada a comp licações oculares. Devido ao quadro avançado, a episclerite terá resolutividade após a remoção do CEIO e resolução da endoftalmite, neste caso, provavelmente os quadros se correlacionam (LIANG Y, et al., 2021).

O prognóstico dos traumas oculares está relacionado com muitas variáveis que podem ser responsáveis pela acuidade visual final. A massa do CEIO é um fator prognóstico, pois o aumento da massa do CE está associado à lesão do segmento posterior, impacto na retina com descolamento e complicações crescentes. O tamanho do CEIO também pode desempenhar um papel na acuidade visual pós-operatória. Devido à sua energia cinética mais alta, um CEIO maior tem mais probabilidade de causar danos graves. A acuidade visual também é um fator de prognóstico importante para o resultado final (LOPORCHIO D, et al., 2016; ZHANG Y, et al., 2011).

A acuidade visual inicial até certo ponto pode ser representativa da gravidade do dano ocular após o trauma (KHATRI A, et al., 2021). O prognóstico visual, no caso, é bastante variável, e está diretamente associado à permanência do CE no olho por um longo período. A paciente foi encaminhada para cirurgia, com indicação de transplante de córnea, facectomia, vitrectomia posterior e retirada de corpo estranho OE. Assim, é de múltipla importância a busca pela ampla propriedade teórico-prático e das formas de manejo do caso em questão.

A suspeita de CEIO deve ser investigada em todos os pacientes com trauma penetrante. Este estudo permitiu a identificação do CEIO com característica metálica no segmento posterior do OE. Ainda possibilitou apontar danos relacionados com a lesão da córnea, ruptura da íris e opacificação do cristalino, além de proporcionar informações que permitiram programar o tratamento cirúrgico das estruturas lesionadas, tanto do segmento anterior quanto posterior. Para preservar os olhos dos pacientes que sof reram traumatismos com CEIO, deve-se sempre antecipar ao tratamento com pesquisas por imagens apropriadas que permitam a correta localização e dimensionamento do CE, para assim, abalizar apropriado programa cirúrgico, de forma que o dano à visão seja amortizado. 


\section{REFERÊNCIAS}

1. BHAGAT N, et al. Post-traumatic Infectious Endophthalmitis. Surv Ophthalmol ., 2011;56(3):214-51.

2. BOURKE L, et al. Clinical outcomes and epidemiology of intraocular foreign body injuries in Cork University Hospital, Ireland:an 11-year review. Ir J Med Sci, 2021;1225-1230.

3. CHORAGIEWICZ T, et al. Surgical treatment of open globe trauma complicated with the presence of an intraocular foreign body. Klin Oczna, 2015; 117(1):5-8.

4. KHATRI A, et al. Ophthalmic Trauma Correlation Matrix (OTCM): a potential novel tool for evaluation of concomitant ocular tissue damage in open globe injuries. Gra efes Arch Clin Exp Ophthalmol., 2021.

5. KAUSHIK S, et al. Occult intraocular foreign body: ultrasound biomicroscopy holds the key. Int Ophthalmol., 2008; 28(1): 71-3.

6. KUHN F, et al. The ocular trauma score (OTS). Ophthalmol Clin N Am ., 2002; 15(2): 163-165.

7. LASSANCE L, et al. Fibrocyte migration, differentiation and apoptosis during the corneal wound healing response to injury. Exp. Eye Res., 2018;170: 177-187.

8. LIANG Y, et al. Intraocular Foreign Bodies: Clinical Characteristics and Factors Affecting Visual Outcome. J Ophthalmol., 2021:9933403.

9. LIU CC, et al. Epidemiology and clinical outcome of intraocular foreign bodies in Hong Kong: a 13 -year review. Int Ophthalmol., 2017,37(1):55-61.

10. LOPORCHIO D, et al. Intraocular foreign bodies: A review. Surv Ophthalmol., 2016;61 (5):582-96.

11. MARINO GK, et al. Epithelial basement membrane injury and regeneration modulates corneal fibrosis after pseudomonas corneal ulcers in rabbits. Experimental Eye Research, 2017;161: 101-105.

12. RATHOD R, MIELER WF. An update on the management of intraocular foreign bodies. Retin Physician, 2011; 8: 5255.

13. RONG AJ, et al. Multimodal imaging features of intraocular foreign bodies. Semin Ophthalmol. , 2019; 34(7-8): 518532.

14. SALAMA AMR, et al. Is this a worrisome red eye? Episcleritis in the primary care setting. Journal of community hospital internal medicine perspectives, 2018;8(1): 46-48.

15. SHAH M, et al. Controversies in traumatic cataract classification and management: a review. Can J Ophthalmol., 2013; 48(4): 251-8.

16. TORRICELLI AAM, et al. Transmission electron microscopy analysis of epithelial basement membrane repair in rabbit corneas with haze. Invest. Ophth. Vis. Sci., 2013;54: 4026-33.

17. UNVER YB, et al. Ocular trauma score in open-globe injuries. J Trauma, 2009;66(4): 1030-2.

18. UPSHAW JE, et al. Ocular foreign bodies in children. Pediatr Emerg Care, 2008;24(6): 409-14.

19. WILSON SE. Corneal wound healing. Exp Eye Res., 2020; 197.

20. YEH S, et al. Current trends in the management of intraocular foreign bodies. Curr Opin Ophthalmol. , 2008; 19(3): 22533.

21. ZHANG Y, et al. Intraocular foreign bodies in China: clinical characteristics, prognostic factors, and visual outcomes in 1,421 eyes. Am J Ophthalmol., 2011;152(1):66-73.

22. ZHU L, et al. Ocular trauma score in siderosis bulbi with retained intraocular foreign body. Medicine (Baltimore), 2015; 94(39): e1533. 